\title{
Etnografía cuantitativa Revitalización lingüística y difusión de las tecnologías digitales en municipios de Oaxaca, México*
}

\author{
Quantitative ethnography \\ Linguistic revitalization and the spread of digital technology \\ in Oaxaca, Mexico's municipalities
}

\author{
MANUEL GERARDO CHÁVEZ ÁNGELES** \\ JOSELITO FERNÁNDEZ TAPIA***
}

\begin{abstract}
This article's objective is to analyze the relationship between indigenous languages and internet access in Oaxaca. Using quantitative methodology, we estimate the probability for Oaxacan municipalities to have internet access. We calculate the correlation between being an indigenous language-speaker and social media user. The results of the analysis present a positive correlation between the number of mobile phones in a municipality and internet access. Likewise, social media usage is correlated positively with being an indigenous language-speaker.
\end{abstract}

Key words: e-government, social inclusion, social networks, digital divide, public sphere

\begin{abstract}
Resumen
El objetivo del artículo es analizar la relación entre las lenguas originarias y el acceso a internet en Oaxaca. Se emplea una metodología cuantitativa con la que se estima la probabilidad de que los municipios de ese estado tengan acceso a internet. Se calcula la correlación entre ser hablante de una lengua originaria y usar los medios sociales. Los resultados del análisis presentan una correlación positiva entre el número de teléfonos móviles en un municipio y el acceso a internet. Asimismo, el uso de medios sociales digitales está correlacionado de forma positiva con ser hablante de lengua originaria. Palabras clave: gobierno electrónico, inclusión social, redes sociales, brecha digital, esfera pública
\end{abstract}

* Artículo recibido el 18/06/19 y aceptado el 07/11/19.

** Universidad de la Sierra Sur. Guillermo Rojas Mijangos s/n, col. Ciudad Universitaria, 70800, Miahuatlán de Porfirio Díaz, Oaxaca <manuelchavezangeles@hotmail.com>. ORCID: https: / / orcid.org/0000-0002-4287-6532.

*** Universidad de la Sierra Sur. Guillermo Rojas Mijangos s/n, col. Ciudad Universitaria, 70800, Miahuatlán de Porfirio Díaz, Oaxaca <joseft100@hotmail.com>. orcID: https: / / orcid.org/0000-0003-1847-686X. 


\section{Introducción Esfera pública e internet}

$\mathrm{E}$ $\mathrm{n}$ los últimos años se ha discutido la importancia de fusionar las técnicas cuantitativas y cualitativas de investigación para comprender los fenómenos culturales de la era digital. A dicho método se le ha llamado etnografía cuantitativa (Shaffer, 2017). Asimismo, desde la ciencia política se ha discutido la pertinencia de utilizar métodos etnográficos para el estudio de las relaciones de poder (Wedeen, 2010; Brodkin, 2017). El presente artículo presenta un análisis etnográfico cuantitativo sobre la adopción de tecnologías digitales en la esfera pública cultural del estado de Oaxaca, en México. Se utilizan técnicas de la ciencia de datos, en particular regresiones multivariadas, para estudiar la relación entre el acceso a internet, el uso de medios sociales digitales y variables culturales, entre estas últimas el bilingüismo del español y lenguas originarias.

Sobre la esfera pública cultural, el filósofo alemán Jürgen Habermas (1981) analizó de qué manera las opiniones de los ciudadanos evolucionaron hacia lo que ahora conocemos como opinión pública. La esfera pública de la sociedad civil europea del siglo xviII, en especial en Inglaterra y Francia, fue el campo fértil para el surgimiento de la prensa masiva. Dicha esfera pública permite la participación política de las masas. Como un prototipo, los cafés de Londres y París eran lugares donde se intercambiaban con libertad las noticias y opiniones, principalmente entre varones de distintas clases sociales. La prensa escrita representó en gran medida la transición entre el feudalismo y el capitalismo, al darle voz y presencia a una naciente burguesía y al permitir a las clases menos favorecidas exigir los mismos derechos que los de sus patrones (McGuigan, 2005; Boeder 2005).

Eventualmente surgieron espacios menos politizados en favor de una industria comercial más dedicada al entretenimiento, que en algunos casos vulnera la lucha por los derechos políticos y sociales de las clases populares. Habermas (1981) también estudia de qué manera, a mediados del siglo xx, la libertad de prensa y el debate abierto se vieron diluidos y distorsionados por consideraciones comerciales y de relaciones públicas, mientras que, por su parte, el Estado de bienestar incorporaba muchas de las demandas más radicales. Así, las masas se convirtieron en consumidores divertidos e indiferentes a los temas políticos más apremiantes.

El surgimiento de los medios electrónicos complicó más este panorama, incluso dificultó distinguir entre ficción y realidad. No obstante, los medios electrónicos han sido cada vez más ubicuos y descentralizados.
En estos espacios el ideal de la esfera pública como arena de deliberación democrática puede verse empañado por el populismo poco crítico y el radicalismo (McGuigan, 2005; Boeder 2005). Asimismo, puede verse afectado por la brecha digital y, consecuentemente, por la ausencia de inclusión digital en amplios sectores sociales, que no pueden ser parte de la nueva opinión pública asociada a internet, cuyo impacto en lo social y político es importante.

En este sentido, la inclusión digital tiene un doble efecto en la esfera pública cultural. Por un lado, democratiza las opiniones llevando los "cafés" a internet y permitiendo un intercambio de información más fluido entre personas de distintos ingresos, clases y etnicidades. Por el otro, permite la industrialización y comercialización de la cultura, que se convierte en fuente de entretenimiento de gran trascendencia. En estos procesos interviene la interculturalidad, aunque también es amenazada por la homogeneización global y la multiculturalidad irreconciliable, no sólo en lo local sino en lo nacional y lo global.

En el caso de Oaxaca, la etnicidad es un factor fundamental en la construcción de la esfera pública cultural. Habermas (1981, 1996 y 1999) no aborda el tema étnico en sus estudios; es probable que esto se deba al fantasma del nazismo. Sin embargo, en el caso de México la interculturalidad cumple un relevante papel para entender el efecto de las tecnologías digitales en la esfera pública cultural, en particular en escenarios multiétnicos como los que estudia este artículo.

Es menester señalar la importancia de la pluralidad y las diferencias para construir sociedades interculturales. La ciudadanía democrática desde la interculturalidad debe ser compartida. Es necesario implementar una democracia para la diversidad, que considere la etnicidad y la diversidad cultural en el marco de un nuevo pacto social incluyente (Bilbeny, 1999 y 2002). Ciudadanía y democracia pluralistas que respondan a una sociedad culturalmente inteligente. La cultura es componente de un modelo social de territorio inteligente en la sociedad del conocimiento (Mitxelena Lakarra, 2015), de ciudades culturalmente inteligentes (Innerarity, 2015) y de una democracia del conocimiento que vincula política, ciudadanía, participación e información, en torno al conocimiento (Innerarity, 2011). A la vez, hay que tener en cuenta que el internet no es sólo ni principalmente una tecnología, sino que es una producción cultural (Castells, 2003). Desde estas perspectivas, la e-inclusión cultural y la lengua en particular tienen un lugar preponderante.

De igual modo, la esfera pública es un factor de gran envergadura en la revitalización lingüística. Elementos 
lingüísticos de la esfera pública de una comunidad, como la señalética, los toponímicos, los medios de comunicación masiva y el e-gobierno son determinantes en la revitalización lingüística. Al respecto, Ghil'ad Zuckermann y Rob Amery (2015) crearon el esquema diamante de revitalización lingüística (LaRD por sus siglas en inglés) para analizar y desarrollar programas de revitalización lingüística. Entre los cuatro componentes del LaRD se encuentran: $a$ ) las comunidades de hablantes; $b$ ) el sistema educativo y los métodos de aprendizaje; $c$ ) la investigación lingüística; $d$ ) y la esfera pública.

En el presente artículo, se utiliza un modelo probit para estimar la probabilidad de que un municipio de Oaxaca tenga acceso a internet. Esa probabilidad guarda una correlación positiva con el porcentaje de la población con teléfonos móviles. Esto permite inferir que las tecnologías móviles son las determinantes esenciales de la e-inclusión. Por su parte, al hacer un análisis logit con datos etnográficos recabados para este estudio, se encuentra una correlación positiva y estadísticamente significativa entre ser hablante de lengua originaria y hacer uso de los medios sociales digitales, en específico tener una cuenta de Twitter, YouTube o Google.

\section{La revitalización lingüística en México}

En México, la revitalización lingüística de los pueblos originarios es resultado de los movimientos por los derechos de autodeterminación cultural de los pueblos indígenas. En 2001 se reformó el artículo segundo de la Constitución Política reconociendo la composición pluricultural del país sustentada en sus pueblos indígenas. Ese artículo establece que "los pueblos indígenas son aquellos que descienden de poblaciones que habitaban en el territorio actual del país al iniciarse la colonización y que conservan sus propias instituciones sociales, económicas, culturales y políticas, o parte de ellas".

Un derecho de los pueblos y las comunidades indígenas que se reconoce es el de la libre determinación y, en consecuencia, el de la autonomía para preservar y enriquecer sus lenguas, conocimientos y todos los elementos que constituyen su cultura e identidad.

El artículo segundo constitucional también especifica que en todos los juicios y procedimientos en que sean parte los pueblos indígenas o sus integrantes debe garantizarse el acceso pleno a la jurisdicción del Estado y, para tal fin, deben tenerse en cuenta las costumbres y especificidades culturales de dichos pueblos, considerando, entre otros, el derecho a ser asistidos por intérpretes y defensores que tengan conocimiento de su lengua y cultura (Instituto Nacional de Lenguas Indígenas, 2008).

En 2003 el Congreso de la Unión aprobó la Ley General de Derechos Lingüísticos de los Pueblos Indígenas (LGDLPI). Tras el reconocimiento constitucional de las lenguas indígenas como lenguas oficiales, la LGDLPI creó el Instituto Nacional de Lenguas Indígenas (Inali) con los siguientes objetivos:

- Promover el fortalecimiento, preservación y desarrollo de las lenguas indígenas que se hablan en el territorio nacional.

- Promover el fortalecimiento, preservación y desarrollo del conocimiento y disfrute de la riqueza cultural de la nación.

- Asesorar a los tres órdenes de gobierno para articular las políticas públicas necesarias en la materia.

En el Catálogo de las Lenguas Indígenas Nacionales. Variantes lingüísticas de México con sus autodenominaciones y referencias geoestadísticas (Inali, 2008) se reconoce la existencia de 364 variantes lingüísticas, conformadas en 68 agrupaciones lingüísticas y 11 familias lingüísticas.

En términos de uso de tecnologías digitales para la revitalización lingüística, el Inali cuenta con una página de internet ${ }^{1}$ donde se encuentra la legislación en materia de derechos lingüísticos junto con algunos recursos como el Acervo de Lenguas Indígenas Nacionales (ALIN), un acervo documental abierto, físico y digital que contiene datos de lenguas indígenas nacionales, como vocabularios, frases y narraciones en formato de audio y video, y que está disponible electrónicamente; ${ }^{2}$ su finalidad es preservar datos de lenguas que están en alto riesgo de desaparecer, y tener a disposición material lingüístico para proyectos de investigación y conservación de lenguas.

Según datos abiertos del propio Inali, en Oaxaca se hablan unas 41 variantes de lenguas indígenas; suman 1205886 los hablantes de una lengua originaria, alrededor de una tercera parte de la población estatal, y $16.33 \%$ de los hablantes a nivel nacional.

Entre las lenguas con mayor número de hablantes en la entidad oaxaqueña se encuentran el zapoteco, con 405307; mixteco, con 266372; mazateco,

\footnotetext{
1 https://www.inali.gob.mx/.

2 http://alin.inali.gob.mx/xmlui/.
} 
con 179299; mixe, con 114833, y chinanteco, con 107838. Hay varias lenguas con un muy reducido número de hablantes que están en peligro de desaparecer, entre ellas el akateki, con un hablante; el chichimeco jonaz, con un hablante también; el chontal, con cinco hablantes. Sin embargo, hay que reconocer que algunas de estas lenguas no son originarias de Oaxaca $;^{3}$ no obstante, se requiere su revitalización.

\section{La brecha digital y la pobreza digital en el estado de Oaxaca}

La noción de brecha digital se genera a partir de los análisis sobre la incidencia de las tecnologías de la información y la comunicación (TIC) en la estructura social y el mundo del trabajo. Se refiere a las disparidades en la adopción de las Tic entre los distintos estratos socioeconómicos en el interior de los países, o entre ellos; pero también entre individuos, familias y distintas unidades territoriales o regiones. Implica además su apropiación, uso y calidad de uso, es decir, saber utilizarlas y obtener rentabilidad de ellas (Olarte Encabo, 2017). Es decir, las desigualdades de acceso a las habilidades digitales y la falta de capacidades para aprovecharlos.

La pobreza digital, asociada a la relación ofertademanda, es entendida como la carencia de conectividad, comunicación, información, así como de capacidades digitales, oportunidades y beneficios que otorgan las TIC, sea por falta de acceso o de conocimiento (Barrantes, 2009):

a) Personas con bajos ingresos económicos que no tienen acceso y uso de las TIC; que tienen capacidades digitales mínimas, pero no tienen oferta o no las demandan.

b) Aquellos que no son económicamente pobres, pero que no demandan las tecnologías digitales porque no tienen conocimiento ni desarrollo de las habilidades digitales.

En Oaxaca, Coria et al. (2011) analizan diversas alternativas para reducir la brecha digital en el estado; entre ellas la responsabilidad social de las grandes empresas de telecomunicaciones con base en las concesiones otorgadas para su operación; los planes del extinto Instituto de Desarrollo de Tecnologías de la Información (IDTI) de Oaxaca para reducir la brecha digital; la posibilidad de otorgar estímulos a empresas privadas de telecomunicaciones, además de crear nue- vas empresas gubernamentales o reorientar algunas para ofrecer servicios de telefonía y acceso a internet, y las experiencias de microtelcos (microempresas de telecomunicaciones) en América Latina.

Por su parte, Chávez-Ángeles y Sánchez-Medina (2013) definen la brecha digital no como un problema meramente tecnológico, sino como un fenómeno social que es parte de un conjunto más amplio de inequidades sociales. Auxiliándose del marco de análisis y desarrollo institucional (que tiene en cuenta las características físicas, los atributos de la comunidad y los arreglos institucionales) y su influencia en la arena de acción (compuesta por situaciones y actores) consideran los patrones de interacción, los cuales deben tener criterios de evaluación de resultados y, junto con la teoría de juegos, exponen el comportamiento de los actores en términos de la maximización de beneficios. Para lograrlo deben realizar la búsqueda de los equilibrios de Nash, que incluyen el principio de las expectativas racionales y los resultados socialmente óptimos, y observan por qué estos equilibrios no están siendo jugados. En otras palabras, estudian los escenarios en los cuales se logra la cooperación y analizan por qué ésta no se presenta en otras condiciones.

Para analizar los dilemas de las telecomunicaciones en el ámbito rural, asocian la brecha digital con criterios de la pobreza digital (la demanda de tecnologías de la información y la comunicación, los "nuevos comunes"). Con base esto, estudian la carencia de bienes y servicios basados en las TIC y concluyen que los municipios rurales de México enfrentan un alto grado de dependencia de las grandes compañías de telecomunicaciones en el desarrollo de sus políticas de conectividad, debido a la atomización de la demanda de servicios de telecomunicaciones por parte de los municipios. Asimismo, se observa una alta concentración de la oferta de dichos servicios en manos de unos cuantos prestadores de servicios de internet. Por lo tanto, se requiere crear mecanismos de coordinación intermunicipal que permitan invertir de manera conjunta en la conectividad rural. En este sentido es indispensable crear un fondo (o fideicomiso) de conectividad rural en el que los municipios geográficamente aledaños puedan destinar recursos financieros a una bolsa común que les permita atraer la inversión de las grandes empresas de telecomunicaciones a sus regiones. De igual modo, es de gran importancia que los municipios logren consensos sobre el tipo de tecnología que desean instalar, potenciando así las externalidades tecnológicas y de red. La decisión sobre el tipo de TIC (ej. móvil vs. fija) es tan esencial como

3 https: / / www.inali.gob.mx/. 
la de inversión en ті о т, debido a que la red genera beneficios colectivos que justifican un subsidio equivalente al valor de las externalidades.

La permanencia de la brecha digital y el hecho de que los municipios instrumenten políticas para disminuirla o eliminarla demuestran que el acceso a las TIC se traduce en desigualdad de posibilidades de acceso a la información y el conocimiento; no es sólo una cuestión tecnológica, sino también de aspectos socioeconómicos que inciden de forma negativa en la dimensión de la pobreza y la exclusión social (Olarte Encabo, 2017). Adicionalmente, también abarca aspectos políticos y socioculturales y, por lo tanto, de inclusión o exclusión cultural.

\section{Metodología y datos: etnografía cuantitativa}

Una característica del nuevo milenio es que las ciencias en general enfrentan problemas cada vez más complejos: la ecología y el medio ambiente; las estrellas y galaxias; los átomos y electrones; la célula y sus moléculas; el microbioma humano; la economía global; el cerebro humano. Esa complejidad no puede ser resuelta por una sola disciplina y método, se requiere un replanteamiento o innovación metodológica.

Estos retos metodológicos son acompañados de un alud de información proveniente de las nuevas tecnologías digitales. Los macrodatos o inteligencia de datos (el big data) y la ciencia de datos se vuelven una necesidad. No obstante, la construcción de significados colectivos se encuentra todavía lejos de las capacidades computacionales más sofisticadas de la inteligencia artificial. Hasta nuestros días, no existe sistema computacional capaz de simular y predecir la construcción semántica hecha por los humanos de manera colectiva. Los datos por sí solos no tienen ningún significado. Únicamente cuando se les asigna un sentido semántico es que se transforman en información, y entonces podemos construir nuevos conocimientos. La ciencia de datos proporciona herramientas para encontrar dichos significados (Shaffer, 2017).

Así, la necesidad de realizar análisis cuantitativo se deriva de esta nueva realidad tecnológica, en la que estamos rodeados de números a los cuales dotamos de significado y convertimos en información. Una disciplina útil para analizar el significado de esta "información" es la etnografía: una ciencia que estudia la cultura. Si bien las computadoras generan datos y la estadística los analiza, la cultura les otorga un cierto significado que la etnografía nos ayuda a desentrañar. La cultura importa porque los seres hu- manos traficamos símbolos en nuestras acciones, en nuestra oralidad y en nuestros textos. Las cosas que la gente hace, dice y escribe son interpretadas por otros que comparten su cultura. La cultura es: $a$ ) la manera como las personas entienden las cosas, b) la conexión entre las cosas, c) la conexión entre las cosas y las personas y, $d$ ) cómo las personas se conectan entre ellas. La etnografía es la ciencia que busca comprender dichas interpretaciones y conexiones (Shaffer, 2017), de allí su trascendencia y utilidad.

Los datos que utilizamos tienen dos fuentes: la primera, los datos del Instituto Nacional de Estadística y Geografía (INEGI), representativos a nivel nacional. De éstos se analizan los correspondientes a los municipios de Oaxaca. Simultáneamente, se aplicó una encuesta a usuarios de internet utilizando técnicas etnográficas para el estudio de difusión de dispositivos móviles. Debido a la complejidad de este estudio y la diversidad multicultural del estado de Oaxaca, se realizó un muestreo intencionado con los siguientes criterios: a) se seleccionó un municipio por región; b) municipios cuyo gobierno municipal tuviera sitio web; c) municipios de relevancia económica, industrial y/o turística. Con estos criterios, durante 2015 se efectuó trabajo de campo en seis municipios: Huajuapan de León, Juchitán de Zaragoza, Salina Cruz, Santa María Huatulco, San Juan Bautista Tuxtepec, San Pedro Mixtepec. Se hicieron 277 encuestas

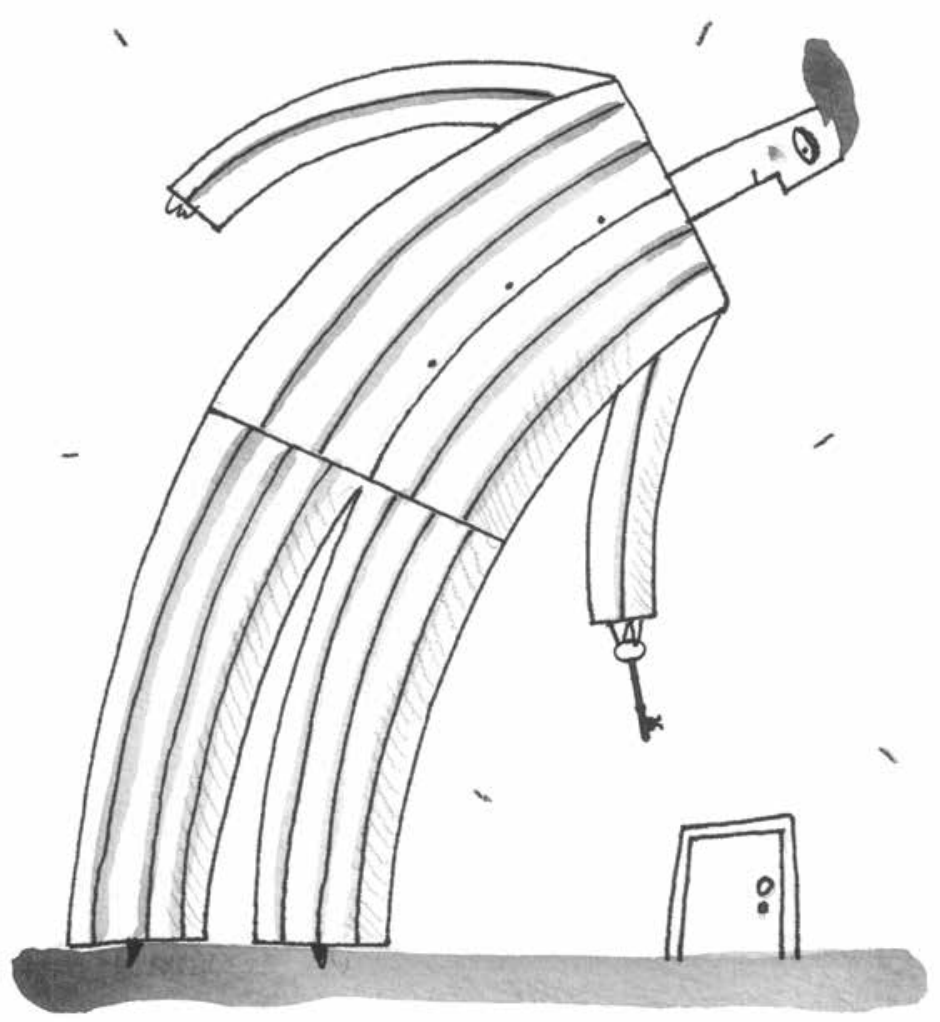


con población usuaria de Tic, tomando un muestreo de máxima variación para tratar de cubrir el espectro de personas susceptibles de usar los aparatos móviles. Se incluyeron preguntas en las que los encuestados dieron sus puntos de vista de cómo las TIC pueden potencializar el desarrollo humano y la democracia. Los instrumentos de recolección de información fueron diseñados ad hoc para el proyecto, y fueron afinados mediante pruebas piloto antes de hacer el levantamiento final, de manera que respondieran a los objetivos de la investigación.

Se utilizan dos tipos de modelos de regresión binaria: probit y logit, donde las variables dependientes son de naturaleza cualitativa.

En general los modelos logit y probit se estiman con un modelo como el siguiente:

$$
P_{i}=E(Y=1 \mid X)=\beta_{1}+\beta_{2}+\beta_{3}+\ldots+\varepsilon
$$

Donde $\mathrm{X}$ es un vector de variables sobre características de los usuarios y $\mathrm{Y}=1$ significa que: a) el municipio tiene acceso a internet; b) los entrevistados tienen cuenta en Twitter; c) los entrevistados tienen cuenta en YouTube; y d) los entrevistados tienen cuenta en Google.

En la primera regresión se estima la probabilidad de que el municipio tenga acceso a internet público utilizando los datos del Censo Nacional de Gobiernos Municipales y Delegacionales 2015 del INEGr. Se utilizan los datos para los 570 municipios del estado de Oaxaca. El censo tiene un módulo de gobierno electrónico que hace los datos muy útiles para este tipo de análisis.

En la regresión logit se utilizó la encuesta etnográfica levantada para el proyecto. Con esta muestra se corrieron tres regresiones multivariadas utilizando como variables dependientes tres proxys sobre el uso de medios sociales (utiliza Twitter, Youtube, Google) y se trató de encontrar las correlaciones con el aspecto de bilingüismo de español y lengua originaria.

\section{Resultados}

El diagrama 1 muestra la dispersión de la probabilidad de que cada municipio en el estado de Oaxaca tenga acceso a internet contra el porcentaje de teléfonos celulares en ese municipio. La probabilidad fue calculada utilizando una base de datos de los 570 municipios de Oaxaca por medio de un modelo probit, como se describió en la sección anterior.

En el cuadro 1 se exponen los resultados del modelo con el que se calculó dicha probabilidad. Entre las variables con significancia estadística en el modelo están: el porcentaje de la población con teléfonos móviles cuyo coeficiente es de 0.007, con 90\% de confianza; si tiene piso de tierra tiene un coeficiente de -0.009, y su nivel de confianza es de $95 \%$; el porcentaje de viviendas con radio en el municipio tiene un coeficiente de 0.013 , con $95 \%$ de confianza; el porcentaje de viviendas con televisión en el municipio es de 0.0046 con 95\% de confianza; el de viviendas con una computadora personal es de 0.031 , con un nivel de confianza de $95 \%$.

Se utilizan más variables que, aunque no reportan significancia estadística, son útiles como variables de control, por ejemplo los porcentajes de: dormitorios, de niños menores de dos años, de viviendas con agua potable, con drenaje, con refrigerador, con lavadora, con automóvil y de viviendas con teléfono fijo.

Diagrama 1

Dispersión de la probabilidad de que un municipio tenga internet vs. porcentaje de celulares en el municipio para Oaxaca

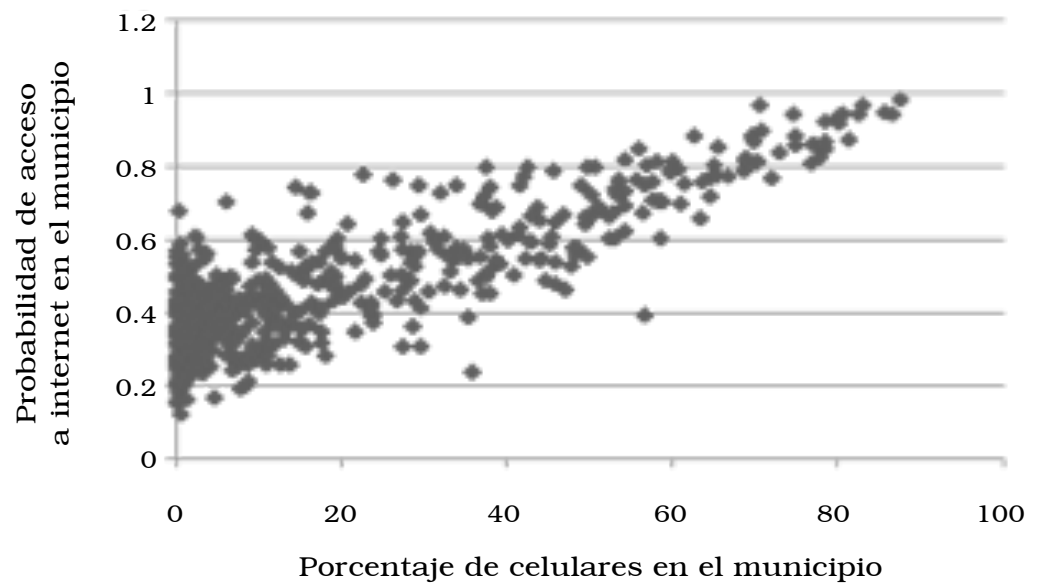

Fuente: Elaboración propia con datos del INEGI. 


\section{Cuadro 1 \\ Modelo probit de acceso a internet en los municipios de Oaxaca}

\begin{tabular}{lcc}
\hline \multicolumn{1}{c}{ Variables independientes } & $\begin{array}{c}\text { Variable dependiente: } \\
\text { acceso a internet } \\
\text { Coeficiente }\end{array}$ & Error estándar \\
\hline Porcentaje de teléfonos móviles & $0.0079693^{*}$ & 0.0051891 \\
Porcentaje de piso de tierra & $-0.0091829^{* *}$ & 0.0047188 \\
Porcentaje de dormitorios & -0.1370679 & 0.101124 \\
Porcentaje de niños menores de dos años & -0.1326148 & 0.1017753 \\
Porcentaje de viviendas con agua potable & -0.0024483 & 0.0026836 \\
Porcentaje de viviendas con drenaje & -0.0028386 & 0.0026066 \\
Porcentaje de viviendas con radio & $0.0131842^{* *}$ & 0.0048168 \\
porcentaje de viviendas con TV & $0.004649^{* *}$ & 0.0067304 \\
Porcentaje de viviendas con refrigerador & 0.0071201 & 0.0065566 \\
Porcentaje de viviendas con lavadora & -0.0040416 & 0.0067414 \\
Porcentaje de viviendas con auto & -0.0127528 & 0.0108299 \\
Porcentaje de viviendas con PC & $0.0318514^{* *}$ & 0.0155336 \\
Porcentaje de viviendas con teléfono & 0.0017795 & 0.0071653 \\
Constante & 12.83556 & 10.04273 \\
\hline
\end{tabular}

* Estadísticamente significativo con 90\% de confianza.

** Estadísticamente significativo con 95\% de confianza.

Fuente: Elaboración propia.

En el cuadro 2 se presentan los resultados de tres regresiones logit utilizando los datos recolectados en campo. Las regresiones incluidas tratan de explicar en qué medida el uso de medios sociales digitales, como Twitter, YouTube y Google está correlacionado con el bilingüismo de español y lengua originaria.

Como puede observarse, en cuanto al uso de Twitter el análisis multivariado reporta un coeficiente de 1.90 , siendo estadísticamente significativo con $95 \%$ de confianza para hablantes de alguna lengua originaria. De igual manera, el modelo reporta un coeficiente de 2.51 para aquellos que son estudiantes, siendo estadísticamente significativo con $95 \%$ de confianza. Es interesante observar que el acceso a internet en casa es una variable que reporta un coeficiente de 1.92 , estadísticamente significativo con 95\% de confianza.

En cuanto a los usuarios de YouTube el modelo reporta un coeficiente positivo para los hablantes de lengua originaria, pero no es estadísticamente significativo. Ser estudiante reporta un coeficiente de 1.08, estadísticamente significativo con $90 \%$ de confianza. De igual forma, el acceso a internet en casa reporta un coeficiente de 1.16, estadísticamente significativo con 95\% de confianza. En este caso, tener acceso a internet en la escuela tiene un coeficiente de 1.88 , estadísticamente significativo con $95 \%$ de confianza.

Para los usuarios de Google el modelo reporta un coeficiente de 1.53, estadísticamente significativo con $90 \%$ de confianza para los hablantes de lengua ori- ginaria. Por su parte, la variable de estudiante tiene un coeficiente de 1.81 , estadísticamente significativo con $95 \%$ de confianza. En este caso, el tener acceso a internet en casa reporta un coeficiente de 0.80 , estadísticamente significativo con $90 \%$ de confianza. Mientras que tener internet en la escuela reporta un coeficiente de 1.67, estadísticamente significativo con 95\% de confianza.

Se utilizan distintas variables de control entre las que se incluyen si tiene teléfono móvil, si es mujer, si tiene empleo, si es ama de casa, si trabaja de forma independiente, si está desempleado, si es servidor público y si tiene acceso a internet en el trabajo. Si bien estas variables no son estadísticamente significativas, son útiles para darle significancia estadística a las variables relevantes del modelo.

\section{Discusión}

En términos de la distribución del acceso a la tecnología y la información, la brecha digital y la pobreza digital son visiones distintas de un mismo problema. Los criterios para definir la brecha digital varían de una comunidad a otra, de un país a otro y de una sociedad a otra. La premisa básica, no obstante, es la diferencia que existe entre individuos y sociedades que tienen acceso a las tecnologías digitales y de telecomunicaciones. También hay otros aspectos 


\section{Cuadro 2}

Uso de redes sociales y telefonía móvil

\begin{tabular}{|c|c|c|c|}
\hline \multirow{2}{*}{ Variables independientes } & \multicolumn{3}{|c|}{ Variables dependientes (logit) } \\
\hline & Twitter & YouTube & Google \\
\hline Móvil & $\begin{array}{l}-0.9321 \\
(0.9656)\end{array}$ & $\begin{array}{l}0.6802 \\
(0.8610)\end{array}$ & $\begin{array}{l}0.6867 \\
(0.8695)\end{array}$ \\
\hline Mujer & $\begin{array}{l}-0.3792 \\
(0.5182)\end{array}$ & $\begin{array}{l}-0.4767 \\
(0.3915)\end{array}$ & $\begin{array}{l}-0.5102 \\
(0.4190)\end{array}$ \\
\hline Bilingüe & $\begin{array}{l}1.9098^{* *} \\
(0.6418)\end{array}$ & $\begin{array}{l}0.828 \\
(0.5838)\end{array}$ & $\begin{array}{l}1.5356^{*} \\
(0.5990)\end{array}$ \\
\hline Estudiante & $\begin{array}{l}2.5108^{* *} \\
(0.9358)\end{array}$ & $\begin{array}{l}1.0881^{*} \\
(0.5642)\end{array}$ & $\begin{array}{l}1.8169 * * \\
(0.5825)\end{array}$ \\
\hline Empleado & $\begin{array}{l}0.7545 \\
(1.0057)\end{array}$ & $\begin{array}{l}1.0111 \\
(0.5738)\end{array}$ & $\begin{array}{l}0.7333 \\
(0.6939)\end{array}$ \\
\hline Ama de casa & $\begin{array}{l}1.7136 \\
(1.0057\end{array}$ & $\begin{array}{l}0.0395 \\
(1.1421)\end{array}$ & -- \\
\hline Independiente & $\begin{array}{l}1.0992 \\
(1.3502)\end{array}$ & -- & $\begin{array}{l}0.3811 \\
(1.1910)\end{array}$ \\
\hline Desempleado & $\begin{array}{l}2.5452 \\
(1.4609)\end{array}$ & $\begin{array}{l}-0.2063 \\
(1.4373)\end{array}$ & -- \\
\hline Servidor público & $\begin{array}{l}2.9708^{*} \\
(1.5049)\end{array}$ & -- & -- \\
\hline Internet en casa & $\begin{array}{l}1.9225^{* *} \\
(0.6644)\end{array}$ & $\begin{array}{l}1.1668^{* *} \\
(0.3982)\end{array}$ & $\begin{array}{l}0.8072 * \\
(0.4236)\end{array}$ \\
\hline Internet en escuela & $\begin{array}{l}-0.2921 \\
(0.6263)\end{array}$ & $\begin{array}{l}1.8888^{* *} \\
(0.4887)\end{array}$ & $\begin{array}{l}1.6718^{* *} \\
(0.4709)\end{array}$ \\
\hline Internet en trabajo & $\begin{array}{l}1.2368 \\
(0.7872)\end{array}$ & $\begin{array}{l}0.4292 \\
(0.5105)\end{array}$ & $\begin{array}{l}0.3715 \\
(0.5912)\end{array}$ \\
\hline Constante & $\begin{array}{l}-4.7248^{* *} \\
(1.182)\end{array}$ & $\begin{array}{l}-3.9285^{* *} \\
(0.9173)\end{array}$ & $\begin{array}{l}-4.2844^{* *} \\
(0.9527)\end{array}$ \\
\hline
\end{tabular}

* Estadísticamente significativo con $90 \%$ de confianza.

** Estadísticamente significativo con 95\% de confianza.

Fuente: Elaboración propia.

determinantes como la educación, la lengua, el género, el ingreso, la edad y la geografía.

Desde esta perspectiva, la brecha digital no es un problema meramente tecnológico, sino un fenómeno social que forma parte de un conjunto más amplio de inequidades sociales. Por su parte, la pobreza digital se encuentra asociada a la demanda y oferta de tecnologías de la información y comunicación y la carencia de ingresos o conocimiento y habilidades digitales. En este sentido, la pobreza digital está determinada por la carencia de bienes y servicios digitales. En los municipios de Oaxaca están presentes tanto la bre- cha como la pobreza digitales; esta última se debe principalmente a la carencia de recursos económicos derivada de la falta de conocimientos y habilidades digitales, pero también hay quienes, sin estar en la pobreza económica y contando con habilidades TIC, son pobres digitales.

Como ya se ha mencionado, los municipios rurales de Oaxaca enfrentan un alto grado de dependencia de las grandes compañías de telecomunicaciones en el desarrollo de sus políticas de conectividad. Por un lado existe una alta atomización de la demanda municipal de servicios de telecomunicaciones, y por el otro hay 
una alta concentración de la oferta de dichos servicios en manos de unos cuantos prestadores de servicios de internet (PSI).

Las grandes empresas sólo invierten en zonas rurales si cuentan con la demanda suficiente para que les sea rentable; en contraparte hay dificultades de coordinación entre municipios (Chávez-Ángeles y Sánchez Medina, 2013). Esta situación es importante porque un buen porcentaje de los hablantes de lenguas originarias en Oaxaca se encuentran en comunidades rurales. Dada su situación de exclusión social y cultural, hay un amplio sector de estas comunidades que no demanda acceso a tecnologías digitales, lo cual es una determinante de la exclusión digital.

En términos del bilingüismo, la interculturalidad es un discurso que ha surgido en los últimos años como respuesta a las reivindicaciones de los nuevos actores indígenas en la esfera pública. Podemos situar este fenómeno en la convergencia de procesos simultáneos de endogénesis, hibridación cultural y comunidad indígena desencadenada por una dinámica de transiciones de lo local a lo regional y de ahí a lo transnacional, que van desde las políticas de inclusión social a las reivindicaciones constitucionales de autonomía (Dietz y Mateos Cortés, 2013). Las tecnologías digitales y el ciberespacio son nuevas arenas donde el uso de las lenguas originarias y la interculturalidad encuentran nuevas formas de expresión, comunicación, integración y disidencia, aún poco estudiadas. Para el caso de Oaxaca, se carece de investigaciones que profundicen el tema.

Internet, los encuentros sociales en las ciudades, la migración hacia Estados Unidos, así como el turismo en el estado, llevan a un permanente intercambio cultural que hace necesario enfoques, políticas y acciones interculturales desde la sociedad y desde el estado. De igual modo, la incorporación de los pobladores indígenas al ciberespacio los pone en contacto con la diversidad de Oaxaca, de México y del mundo contemporáneo. Si a ello se agrega la ausencia de páginas web en las lenguas originarias, incluyendo las estatales, las de la Federación y de los municipios, o el acercamiento del pulso de su identidad por estos medios, el problema se vuelve complejo y se dificulta la inclusión digital.

La inclusión digital cultural, en particular lingüística, es un tema pendiente que no está sujeto a la opinión pública en el ciberespacio, y tampoco existen políticas públicas que la impulsen. No obstante, como señala Bilbeny, es fundamental tener en cuenta las diversas culturas, la pluralidad cultural y las diferencias a partir de las cuales se construyan ciudadanías compartidas, democracias para la diversidad y una ética para la diversidad.

Asimismo, la interculturalidad en el ciberespacio se articula con las ciudades culturalmente inteligentes y hacer de la cultura un elemento de los territorios inteligentes (o no inteligentes), en las sociedades del conocimiento actual, para que también incluya al sector rural y étnico, en este caso de Oaxaca. De igual manera, con la democracia del conocimiento, la que debe incluir a los diferentes si parafraseamos a Bilbeny, o "la inclusión del otro" como se refiere Habermas (1999).

Como consecuencia, desde el gobierno y desde la sociedad se estaría impulsando territorios, regiones, comunidades y ciudades inteligentes, que vinculen tecnología y cultura para lograr la inclusión de todos. Procesos y políticas que estimulen la democracia, no sólo política, sino social, económica, cultural y del conocimiento, de todos los territorios y pueblos. En este sentido, el nuevo pacto social debe incorporar también el ámbito digital, y ahí hay que considerar a los indígenas, sus culturas y sus lenguas.

Siguiendo los cuatro componentes del diamante de revitalización lingüística creado por Zuckermann y Amery (2015), ${ }^{4}$ en las comunidades de hablantes se observa una creciente interconexión con el ciberespacio, haciendo uso de sus lenguas. Sin embargo, aprovechar esta interconexión requiere un impulso social y estatal. Es un reto pendiente.

En el sector educativo, el gobierno también ha promovido la educación intercultural a todos los niveles; en varias universidades han surgido programas de investigación lingüística (véase Dietz y Mateos Cortés, 2013). En el ámbito federal, el gobierno de México ha respondido a las demandas de autodeterminación lingüística de los pueblos originarios con una legislación pertinente y mediante la creación del Inali; en concreto en el componente de la esfera pública, el tema de la revitalización lingüística sigue presente como reivindicación, demanda y necesidad desde los años noventa con la lucha zapatista.

Un caso internacional comparable con el de México es el de Bolivia. En el año 2009, con la aprobación de la Constitución Política del Estado, se declararon idiomas oficiales a los 36 hablados en las diferentes naciones y pueblos indígenas originarios de Bolivia. Así iniciaron los cambios e impulso en la educación, la investigación y la normalización lingüística, como un proceso de inclusión sociocultural y de interculturalidad, que cumple los cuatro componentes del LaRD. No obstante, no son procesos consolidados a plenitud.

\footnotetext{
4 Véase también Zuckermann (2016).
} 
Como parte de sus estrategias de revitalización está la enseñanza en las escuelas y espacios comunitarios de transmisión lingüística llamados nidos bilingües. Estos espacios impulsan la transmisión intergeneracional de una lengua o el aprendizaje natural de una lengua desde la primera infancia.

En otras palabras, los nidos bilingües son espacios de encuentro intergeneracional y comunitario donde se comparten saberes, conocimientos, valores y formas de sentir del mundo, mediante la transmisión oral de los idiomas indígenas originarios y el español. El objetivo es revitalizar las lenguas indígenas transmitiéndolas de manera natural entre generaciones, con especial atención a los niños hasta cuatro años, para quienes se disponen espacios donde puedan escuchar y hablar su idioma, y ser parte viva de sus saberes y conocimientos (Instituto Plurinacional de Estudio de Lenguas y Culturas - IPELC, 2017).

Llevar a las comunidades de hablantes al ciberespacio a través de nidos bilingües digitales, en los que no sólo puedan aprender y transmitir sus conocimientos en las comunidades, sino trascender a la sociedad estatal, nacional y global sería un paso importante de la política, que podría garantizar la revitalización de las lenguas indígenas como parte de un proceso intercultural.

\section{Conclusiones}

En lo relacionado con la metodología, el artículo muestra un estudio de etnografía cuantitativa que realiza un análisis de ciencia de datos. Se busca trascender la separación de los métodos cuantitativos y cualitativos e integrarlos en el análisis. Si bien la evidencia mostrada no es suficiente para encontrar relaciones de causalidad y mucho menos para darle validez externa y generalizar los resultados, las regresiones multivariadas permiten encontrar correlaciones que informan la investigación de manera rigurosa.

Las técnicas aquí mostradas son parte de un herramental mayor que en años recientes se ha aplicado con éxito a los así llamados grandes datos, bases de datos generadas por internet con todo tipo de sensores conocidos como "el internet de las cosas" (IoT). El IoT manipula y analiza enormes cantidades de información y representa un gran reto para la ciencia etnológica.

En cuanto a la difusión y uso de tecnologías móviles, se identificó que la probabilidad de que un municipio tenga internet guarda una correlación positiva y estadísticamente significativa con el número de teléfonos celulares en el municipio, también se estableció que el bilingüismo de español y lenguas originarias tiene una correlación positiva con el uso de medios sociales
(Twitter, YouTube y Google) y, en este campo, la tecnología móvil y el internet juegan un rol fundamental.

$\mathrm{El}$ análisis también permite inferir que el acceso a internet en casa y escuela son importantes determinantes en el uso de redes sociales. En consecuencia, el uso del teléfono móvil e internet, articulado a políticas de interculturalidad permitiría no sólo revitalizar las lenguas sino además ayudaría en la construcción de ciudadanías incluyentes y democráticas.

Entre las recomendaciones más trascendentes está la relevancia de las tecnologías móviles e internet en el abatimiento de la brecha digital y en la revitalización lingüística. El caso de las redes sociales puede ser un espacio creativo para ese fin. Sin embargo, al mismo tiempo, se necesitan políticas que lo impulsen.

La inclusión social suele definirse como el proceso de mejora de los términos en los que grupo e individuos participan en la sociedad y también como el proceso de mejora de las habilidades, oportunidades y dignidad de las personas en desventaja por motivo de su identidad (World Bank, 2013). La e-inclusión sería un proceso de inclusión social mediado por TIC, que considera la apropiación de estas tecnologías y del conocimiento, participación e interculturalidad, con el fin de eliminar las desigualdades y la pobreza digital. En este sentido, la inclusión digital puede definirse como el proceso de mejora de los términos en los que un grupo e individuos participan en la sociedad; también es definida como proceso de mejora de habilidades, oportunidades y dignidad de las personas en desventaja por motivo de identidad.

En México y en el contexto latinoamericano es fundamental pensar en la e-inclusión teniendo en cuenta a las minorías lingüísticas, de manera que sus derechos culturales sean respetados en la era de la información y el conocimiento. Esto implica inclusión digital cultural, donde las lenguas originarias cobren la importancia que merecen en relación con las lenguas dominantes como el inglés, el español, el francés, etcétera.

Los efectos de red de las tecnologías digitales contribuyen a enmarcar las lenguas vivas dentro de un reconocimiento, necesario para que sus hablantes sean ciudadanos con plenos derechos lingüísticos y culturales. Por consiguiente, es necesario pensar en términos de e-cultura indígena y e-lengua indígena para construir espacios abiertos a la diversidad cultural que hagan posible la interculturalidad, la pluralidad y la revitalización lingüística.

Sobre cómo impulsar la inclusión digital de hablantes de lengua originaria el artículo muestra evidencia en favor de la difusión de tecnologías móviles. No puede negarse su importancia para la e-cultura indígena y 
e-lengua indígena. Al mismo tiempo, constituyen un espacio para el enriquecimiento cultural de México.

\section{Fuentes}

BARRANTES, ROXANA

2009 "Análisis de la demanda de tic: ¿qué es y cómo medir la pobreza digital?”, en Hernán Galperin y Judith Mariscal (eds.), Pobreza digital. Perspectivas de América Latina y el Caribe, Centro de Investigación y Docencia Económicas, México, pp. 47-84.

Bilbeny, Norbert

1999 Democraciaparaladiversidad,Ariel, Barcelona. BILBENy, NORBERT

2002 Por una causa común, ética para la diversidad, Gedisa, Barcelona.

Boeder, Pieter

2005 "Habermas' heritage: The future of the public sphere in the network society", en First Monday, vol. 10, núm. 9, 5 de septiembre <https: / / firstmonday.org/ojs/index.php/fm/article/ view/1280/1200> [abril de 2017].

Brodkin, Evelyn

2017 "The ethnographic turn in political science: Reflections on the state of the art", en PS: Political Science \& Politics, vol. 50, núm. 1, pp. 131 - 134, DOI: 10.1017 /S1049096516002298.

Castells, Manuel

2003 "La dimensión cultural de internet", ponencia presentada en el ciclo de debates culturales Cultura xxI: ¿nueva economía?, ¿nueva sociedad?, Universidad Abierta de Cataluña/ Instituto de Cultura del Ayuntamiento de Barcelona, Barcelona <http: / /www.uoc.edu / culturaxxi/esp/articles / castells0502/castells 0502.htm> [abril de 2017].

Chávez-Ángeles Manuel Gerardo

y Patricia Soledad Sánchez-Medina

2013 "Information and Communication Technologies (ICTS) as a common-pool resource coordination, competition and the digital divide in eight municipalities of Oaxaca”, en Gestión y Política Pública, núm. especial Electronic Government, pp. 137-171.

Coria-Holguín, Sergio Rafael,

Elsa Apolonia Mendoza-Cortés,

Rafael Martínez-Peláez y Mónica Pérez-Meza

2011 "Perspectivas para reducir la brecha digital en el estado de Oaxaca", en Conciencia Tecnológica, núm. 42, julio-diciembre, pp. 42-48.

Dietz, Gunther y Laura Selene Mateos Cortés

2013 Interculturalidad y educación intercultural en México. Un análisis de los discursos nacionales e internacionales en su impacto en los modelos educativos mexicanos, Secretaría de Educación Pública, México.

HABERMAS, JÜRGEN

1981 Historia y crítica de la opinión pública. La transformación estructural de la vida pública,

HABERMAS, JÜRGEN Gustavo Gili, Barcelona.

1996 "Civil society and the political public sphere", en Between Facts and Norms. Contributions to a Discourse Theory of Law and Democracy (trad. William Rehg), Polity Press, Cambridge, pp. 329-387.
HABERMAS, JÜRGeN

1999 La inclusión del otro. Estudios de teoría política, A \& M Gráfic, Barcelona.

INALI

2008 "Catálogo de las lenguas indígenas nacionales. Variantes lingüísticas de México con sus autodenominaciones y referencias geoestadísticas", en Diario Oficial de la Federación, 14 de enero <https: / /www.inali.gob.mx/pdf/ CLIN_completo.pdf> [mayo de 2017].

INALI

s.f. "Datos abiertos", en Instituto Nacional de Lenguas Indígenas <https://www.inali.gob. $\mathrm{mx} / \mathrm{es} /$ transparencia/datos-abiertos.html> [marzo de 2017].

INEGI

2015 Censo Nacional de Gobiernos Municipales y Delegacionales 2015, Instituto Nacional de Estadística y Geografía, México.

INNERARITY, DANIEL

2011 Democracia del conocimiento. Por una sociedad inteligente, Paidós, Barcelona.

INNERARITY, DANIEL

2015 "Ciudades culturalmente inteligentes", ponencia presentada en la Cumbre de cultura de CGLU. Cultura y ciudades sostenibles, Bilbao, 18-20 de marzo.

Instituto PluRinacional de Estudio de Lenguas

Y Culturas - IPELC

2017 Revitalización lingüística. Nidos bilingües. Documento conceptual, Fondo de las Naciones Unidas para la Infancia, Santa Cruz de la Sierra.

McGuigan, Jim

2005 "The cultural public sphere", en European Journal of Cultural Studies, vol. 8, núm. 4, pp. 427-443, DOI: $10.1177 / 1367549405057827$.

Mitxelena LaKarRa, Miren Lourdes

2015 "La cultura como componente de un modelo social de territorio inteligente en la sociedad del conocimiento. El caso de la política cultural para Tolosa", tesis doctoral, Universidad del País Vasco, Bilbao.

Olarte Encabo, Sofía

2017 "Brecha digital, pobreza y exclusión social", en Temas Laborales, núm. 138, pp. 285-313.

Shaffer, David Williamson

2017 Quantitative ethnography, Cathcart Press, Madison.

WedeEn, LisA

2010 "Reflections on ethnographic work in political science", en Annual Review of Political Science, vol. 13, núm. 1, pp. 255-272, DOI: $10.1146 /$ annurev.polisci.11.052706.123951.

WORLD BANK

2013 Inclusion Matters: The Foundation for Shared Prosperity, World Bank, Washington, DC, DOI: 10.1596/978-1-4648-0010-8.

Zuckermann, GHIL'AD

2016 Sleeping beauties awake: Revivalistics, crossfertilization and wellbeing, Oxford University Press, Nueva York.

ZuCKERMANN, GHIL'AD

Y RoB AMERY

2015 "Language revival: Securing the future of endangered languages, massive", en EdX Open Online Course (моос) <https: / / www.edx.org/ course / language-revival-securing-futureadelaidex-lang101x> [octubre de 2018]. 